\title{
Simulations of MANX, A Practical Six Dimensional Muon Beam Cooling Experiment
}

\author{
Katsuya Yonehara, ${ }^{a, b}$, Kevin Beard ${ }^{c}$, Alex Bogacz ${ }^{c}$, Yaroslav Derbenev ${ }^{c}$ \\ Rolland P. Johnson ${ }^{b}$, Daniel Kaplan ${ }^{a}$, Kevin Paul $^{b}$, Thomas Roberts ${ }^{b}$, \\ allinois Institute of Technology, Chicago, Illinois, U.S.A. \\ ${ }^{\mathrm{b}}$ Muons, Inc., Batavia, Illinois, U.S.A. \\ ${ }^{\mathrm{c}}$ Thomas Jefferson National Accelerator Facility, Newport News, Virginia, U.S.A.
}

\begin{abstract}
A helical cooling channel (HCC) has been proposed to quickly reduce the sixdimensional phase space of muon beams for muon colliders, neutrino factories, and intense muon sources. Simulation studies of the HCC have already verified the use of a channel with solenoidal, and helical magnetic fields of constant amplitude where, by moving to a rotating frame, a $\mathrm{z}$ or time-independent Hamiltonian can be obtained for detailed analytic treatment. In the discussion below, the HCC concept has been extended to have momentum-dependent magnetic field strengths for a six-dimensional Muon collider And Neutrino factory muon beam cooling demonstration eXperiment (MANX). The simulation studies reported here for this experiment have shown that liquid helium can be used as an energy absorber and coolant for superconducting magnetic coils and that the HCC parameters can be varied to reduce the maximum required field magnitudes. These developments make the experiment more practical in that safety requirements are relaxed and the required fields can be achieved with existing technology.
\end{abstract}

Keywords: Muon Beam Cooling, Helical Magnet, Muon Collider

PACS: 29.27.-a, 29.20.-c, 14.60.Ef, 41.85.Lc

\section{INTRODUCTION}

Helical magnets have been used for some time in the control of spin precession in polarized beam devices. One advantage of helical magnets is that, in conjunction with a solenoid field, the fields can provide continuous focusing, dispersion, and correction of chromatic aberration. By using a low $\mathrm{Z}$ material like hydrogen or helium as an absorber in this helical cooling channel (HCC), the six-dimensional phase space of muon beams can be continuously and effectively cooled [1]. The concept of the HCC has been verified by earlier simulation studies [2].

The HCC concept has been extended to have momentum-dependent magnetic field strengths for two new applications. The first is a six-dimensional precooler which would follow a pion decay channel and the second, discussed here, is a sixdimensional Muon Collider And Neutrino Factory muon beam cooling demonstration experiment, MANX [3], which is being designed to follow MICE [4]. We discuss simulations of practical design concepts of the HCC for the MANX experiment in this article. 


\section{A PRACTICAL HCC}

By filling a HCC with liquid helium (LHe), a muon beam can be decelerated and cooled in six-dimensional phase space by ionization energy loss. The HCC magnet parameters must be varied to match the momentum of the beam as it slows down. A muon beam with initial beam momentum of $300 \mathrm{MeV} / \mathrm{c}$ is injected into the HCC. It is possible to produce a large number of muons with this momentum by an $8-\mathrm{GeV}$ proton driver. Approximately $200 \mathrm{MeV} / \mathrm{c}$ of beam momentum is lost in the $\mathrm{HCC}$ in along a 6.4 meters path length through ionization energy loss in LHe, such that the beam momentum is $100 \mathrm{MeV} / \mathrm{c}$ at the end of the HCC. The final beam momentum must be more than $100 \mathrm{MeV} / \mathrm{c}$ to maintain particle stability.

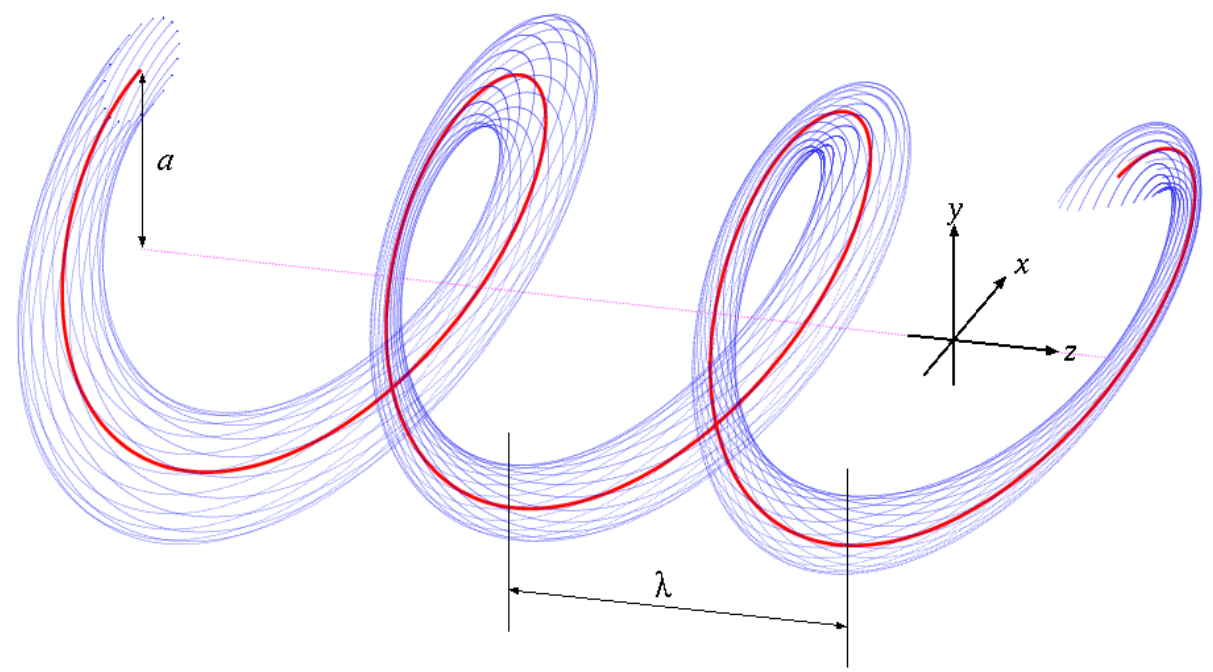

FIGURE 1. Simulated particle tracking in the HCC. The red line is the reference orbit and the blue lines correspond to particles with positions offset from the reference orbit. Here, $a$ is $25 \mathrm{~cm}$.

\section{HCC Magnet Parameters}

From reference [1], the solenoidal and helical magnet parameters for helix period $\lambda$ and helix pitch angle $\arctan (\kappa)$ are given by

$$
\begin{gathered}
B_{z}=\frac{p k(1+q)}{\sqrt{1+\kappa^{2}}}=p_{z} k(1+q), \\
b=\frac{k B_{z}(1-1 /(1+q))}{1+\kappa^{2}}, \\
b^{\prime}=-\frac{g p k^{2}}{\left(1+\kappa^{2}\right)^{3 / 2}}, \\
b^{\prime \prime}=-\frac{b^{\prime}}{2 \hat{D}},
\end{gathered}
$$

where $B_{z}$ is the solenoidal magnet, $b, b^{\prime}$, and $b^{\prime \prime}$ are the helical dipole, quadrupole, and sextupole field components at the reference orbit, $p$ and $p_{z}$ are the reference momentum and its $\mathrm{z}$ component, $k$ is the helix wave number $(=2 \pi / \lambda)$, and $\hat{D}$ is the 
dispersion factor $(=p / a \cdot d a / d p$, where $a$ is the radius of reference orbit), respectively. At the periodic reference orbit, the $q\left(=k_{c} / k-1\right.$, where $k_{c}$ is the cyclotron wave number) and the effective field index $g$ are given by

$$
\begin{gathered}
q=\sqrt{\frac{1+\kappa^{2}-\kappa^{2} \hat{D} / 2}{1+\frac{\kappa^{2} \hat{D} / 2}{1+\kappa^{2}}},} \\
g=\frac{1}{\hat{D}}-\frac{\kappa^{2}+\left(1-\kappa^{2}\right) q}{1+\kappa^{2}},
\end{gathered}
$$

where we have assumed that the cooling decrement in all directions is equal. When $\lambda$ and $\kappa$ are constant at the reference orbit, all magnet parameters are determined by the reference momentum, $p$.

The solution of Maxwell's equations for the helical magnet in a cylindrical coordinate system is given by [5]

$$
b^{(m)}=\left\{\sum_{n=1}^{\infty} n !\left(\frac{2}{n k a}\right)^{n} \frac{I_{n}(n k \rho)}{\rho} \tilde{b}_{n} \cos n \Psi\right\}^{(m)},
$$

where $I_{n}$ is the n-th order modified Bessel function, $\left(\rho, \Psi=\varphi+2 \pi z / \lambda+\varphi_{0}\right)$ is the position in cylindrical coordinates, and $\tilde{b}_{n}$ is the coefficient of the $\mathrm{n}$-th field component. For simplicity, $\tilde{b}_{n}(\mathrm{n}=1,2,3)$ are replaced by $b d, b q$, and $b s$ when the magnet field map is calculated.

The parameters $\lambda$ and $\kappa$ are variables. They can be determined by optimizing the cooling factor in the HCC, constrained by the fact that the field strength of the HCC is determined by them. Therefore, the determination of $\lambda$ and $\kappa$ is essential for the practical design of a HCC. Longer $\lambda$ requires lower field strength. However, this makes for a larger reference radius, and a correspondingly bigger beam pipe radius. Smaller $\kappa$ also lowers the field strength. However, this implies smaller cooling decrements. By tuning both parameters by hand, we found optimum values for $\lambda$ and $\kappa$, which are 2.0 meters and 0.8 , respectively. Figure 2 shows the calculated magnet parameters $(\mathrm{Bz}, b d, b q$, and $b s)$ as a function of $\mathrm{z}$.

\section{SIMULATION RESULTS}

Simulation studies of the HCC for MANX have been done by using two Monte Carlo simulation programs; ICOOL which is developed and maintained by R. Fernow [6], and G4Beamline which is developed and maintained by T. Roberts [7]. Each program gives the same results. Beam tracking is converted in terms of the beam emittance as calculated by the ICOOL post-processing tool ECALC9 [8].

Figure 3 shows the G4Beamline simulation results for the MANX experiment. The emittances are calculated for all particles that survive to $\mathrm{z}=5$. The cooling factors in transverse and longitudinal emittances are about 1.5 at $\mathrm{z}=4$ meters, respectively. Therefore, the six-dimensional cooling factor in this simulation is more than 3 . 

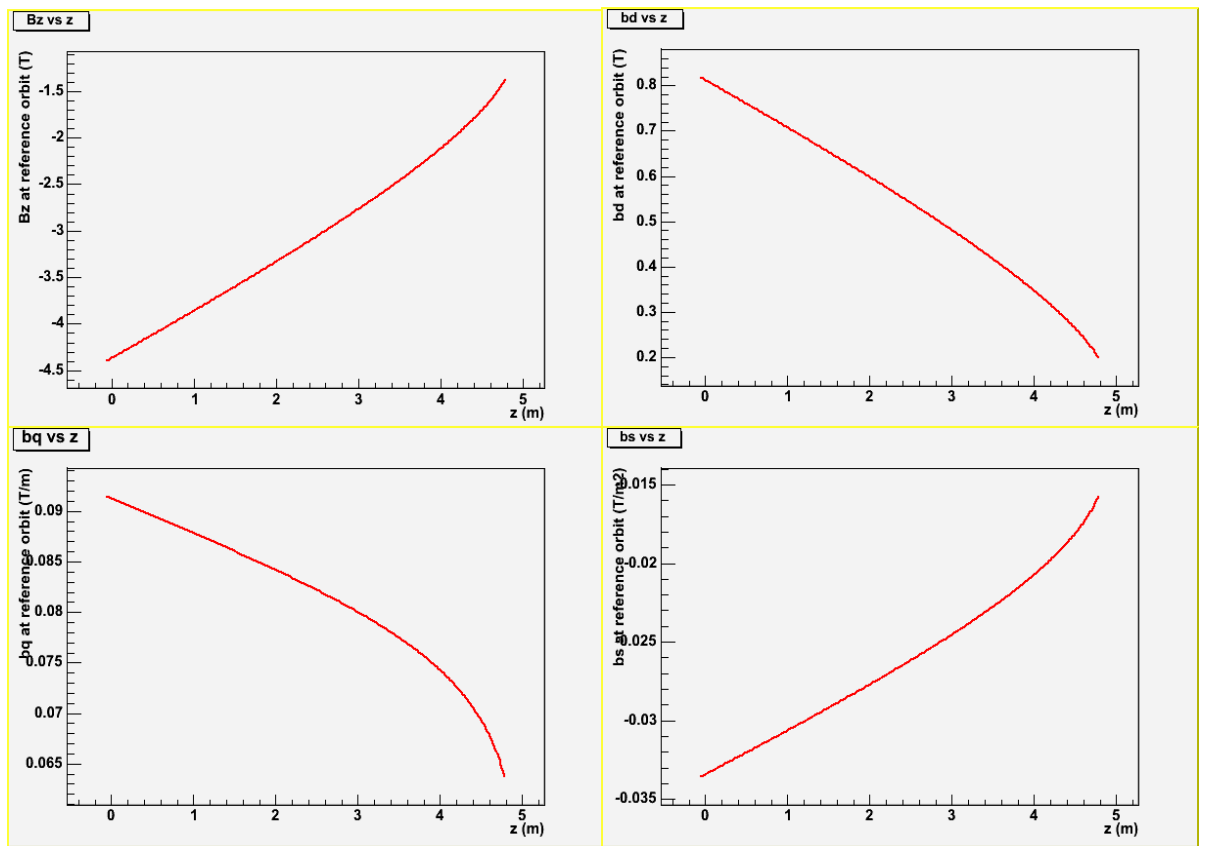

FIGURE 2. Calculated magnet parameters $(\mathrm{Bz}, b d, b q$, and $b s)$ as a function of $\mathrm{z}$. The initial beam momentum is $300 \mathrm{MeV} / \mathrm{c}$, the helix period is 2.0 meters, and the helix pitch angle is 0.8 , respectively. The LHe is used as the absorber.

\section{CONCLUSION}

The first simulation studies in the HCC for the MANX experiment using LHe have shown encouraging results. The use of LHe is only slightly less effective than using LH2 [9]. However, the use of LHe compared to LH2 provides relaxed safety constraints and provides a natural means to cool superconducting magnet coils.

The lower initial momentum, smaller helix pitch angle, and longer helix period compared to those used in earlier studies reduce the maximum field strength to less than $5.5 \mathrm{~T}$ at the superconductor coils. This reduction in the maximum field should be more easily realized in a practical magnet design. 

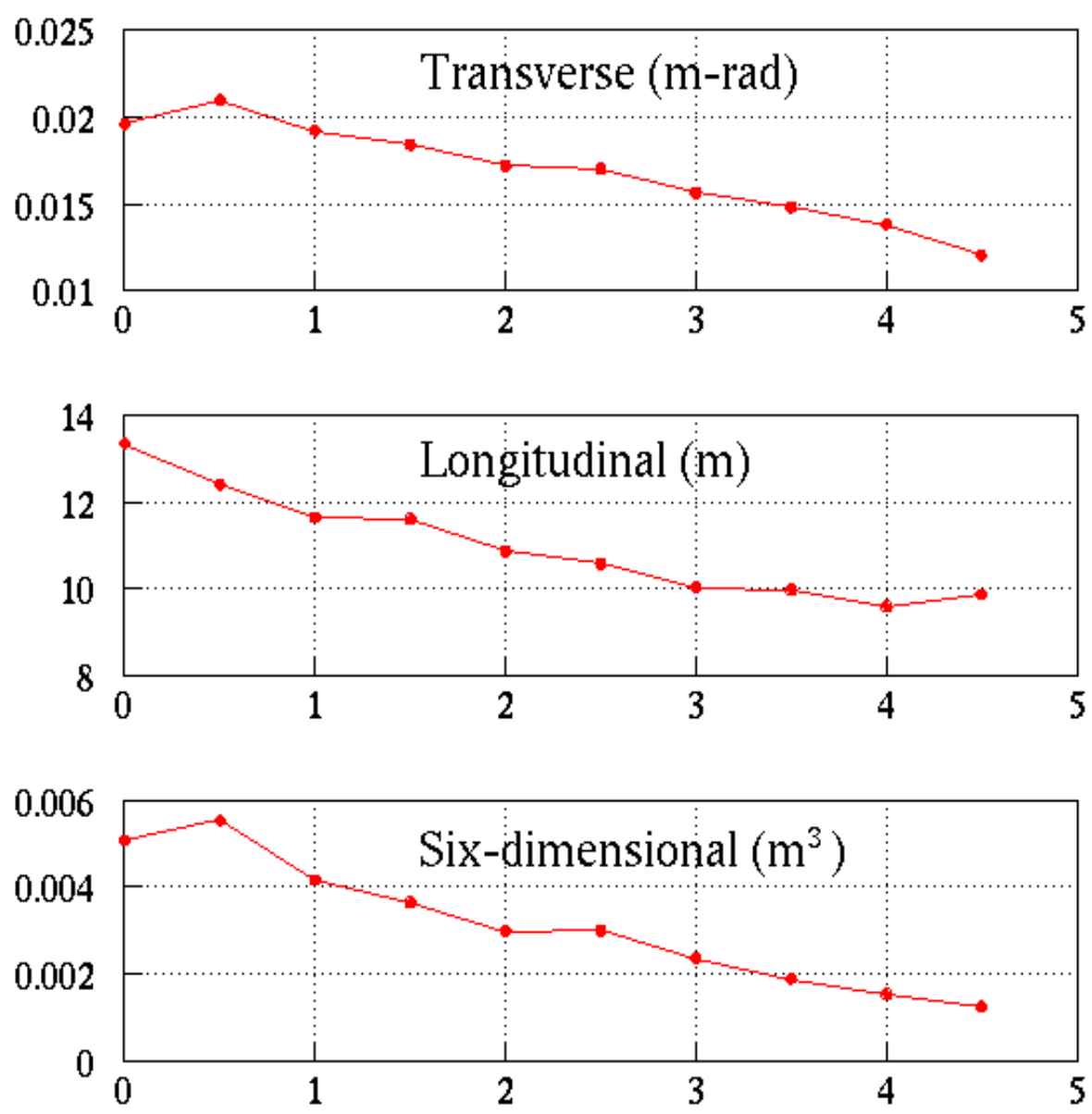

FIGURE 3. Evolution of transverse, longitudinal and six-dimensional emittances as a function of $\mathrm{z}$ as calculated by the G4Beamline simulation program.

\section{REFERENCES}

1. Y. Derbenev and R. P. Johnson, Phys.Rev.STAB 8, 041002, (2005).

2. K. Yonehara et al., to be published in PAC05 proceedings.

3. T. Roberts et al., to be published in PAC05 proceedings.

4. M. A. Cummings et al., to be published in PAC05 proceedings.

5. T. Tominaka et al, Nucl. Instr. and Meth., A459:398, (2001).

6. http://pubweb.bnl.gov/people/fernow/icool/

7. http://www.muonsinc.com/g4beamline.html/

8. http://www-mucool.fnal.gov/mcnotes/public/pdf/muc0280/muc0280.pdf

9. http://snsapp1.sns.ornl.gov/pac05/ROAA005/ROAA005.PDF 